\title{
Calcificaciones metastásicas en un paciente con insuficiencia renal crónica en diálisis peritoneal
}

\author{
Magali Rodríguez Vidal*, Carol Molina Ferrada**
}

*Profesor Asociado, Magíster en Enfermería, Especialista en Diálisis y Trasplante Renal, ** Licenciada en Enfermería Universidad del Bio-Bio, Especialista en Diálisis y Trasplante Renal, Universidad de Concepción, Chile

\section{Resumen}

La enfermedad renal crónica implica un proceso irreversible en la reducción del funcionamiento del tejido renal por lo que la ha llevado a ser un problema de carácter epidémico, donde las complicaciones son devastadoras para la calidad de vida en el paciente, dentro de éstas destacan las calcificaciones metastásicas que se producen por la retención de fósforo, disminución de los niveles de calcio y aumento de la paratohormona. El tratamiento mas adecuado es la paratiroidectomia subtotal con autoimplante, donde la sintomatología y la progresión de las alteraciones del metabolismo mineral se reduce y mejora el estado general del paciente.

Se presenta un caso clínico de una paciente en diálisis peritoneal, con diagnóstico de calcificación metastásica abdominal, a la cual se le realiza una paratiroidectomia - subtotal con autoimplante, donde se observó una buena respuesta con disminución de la calcificación en forma progresiva.

Al evaluar el caso clínico proponemos como enfermeras especialistas en el cuidado del paciente renal, establecer en los centros de diálisis un control semestral por enfermera, independiente de las sesiones de diálisis, donde podamos pesquisar con mayor privacidad y autonomía, las necesidades particulares de cada uno de estos pacientes contribuyendo precozmente a una mejor

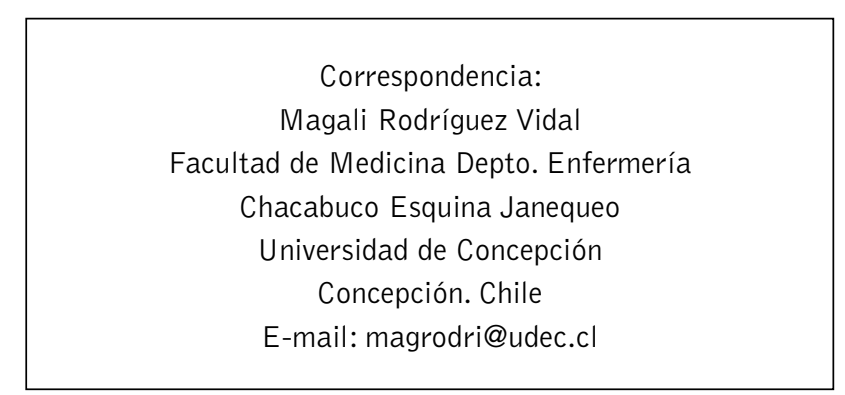

adherencia al tratamiento y retardo progresivo de las complicaciones propias de esta enfermedad.

\section{Introducción}

La enfermedad renal crónica terminal (ERCT) es la disminución progresiva e irreversible del número de nefronas funcionantes, que trae como consecuencia la pérdida de la capacidad de eliminar desechos a través de la orina y mantener el equilibrio acido-base ${ }^{1}$.

En los últimos años esta patología ha experimentado un aumento en todo el mundo, siendo su causa principal la diabetes y en menor medida, hipertensión arterial y glomerulonefritis, las cuales representan un $75 \%$ de los casos totales de ERCT ${ }^{2}$. Por las complicaciones devastadoras que produce, es reconocida como un problema de salud pública global ${ }^{1}$.

El tratamiento de diálisis como sustituto renal, es la técnica que suple parcialmente las funciones renales de excretar agua y solutos y de regular el equilibrio acido-base, no así las funciones endocrinas ${ }^{3}$. Las funciones endocrinas en el paciente en diálisis se encuentran alteradas, de manera sutil y compleja lo que lleva a una dificultad en la interpretación y diagnóstico del déficit y excesos de ellas ${ }^{4}$.

Las alteraciones del metabolismo óseo y mineral son prevalentes en la enfermedad renal crónica y son una causa importante de morbilidad, mortalidad cardiovascular y menor calidad de vida, las cuales son precoces y se inicia con elevación de la hormona paratiroidea y reducción de la vitamina $D$, que progresan con el deterioro de la función renal ${ }^{1}$. En relación a la función de la hormona paratiroidea, esta se encuentra aumentada principalmente por un balance positivo de fósforo e hipocalcemia. La principal consecuencia de esta condición es a nivel óseo (osteodistrofía renal) y cardiovascular. (calcificaciones vasculares, valvulares y calcifilaxis) ${ }^{3}$. 


\section{Calcificaciones metastásicas:}

Las calcificaciones metastásicas fueron descritas por primera vez por Virchow al realizar autopsias en pacientes con enfermedad renal en el año $1855^{2}$. Dentro de los factores relacionados a su aparición, se encuentran la ERCT, hemodiálisis, hiperparatiroidismo, hipervitaminosis $D$, hipercalcemia paraneoplasicas, aumento del producto calcio-fósforo $\left(\mathrm{Ca} \times \mathrm{P}\right.$ ) entre otras ${ }^{5}$. EI hiperparatiroidismo secundario en la falla renal, asociado a hiperfosfemia y elevado producto $\mathrm{Ca} \times \mathrm{P}$, juega un importante rol en los trastornos minerales y óseos que están asociados a eventos cardiovasculares en pacientes con ERCT6.

Las calcificaciones en el paciente con ERCT se caracterizan por el depósito de calcio en tejidos extraesqueléticos. Los depósitos en tejidos blandos y en articulaciones son de fácil identificación; las viscerales son más insidiosas y de mayor peligrosidad, las localizaciones mas frecuentes son: periarticulares, viacerales, vasculares, válvulas cardíacas, arterias coronarias, cerebrales, y otras $^{1,7}$.

Las calcificaciones de arterias coronarias son las de mayor frecuencia, en pacientes sometidos a hemodiálisis en comparación a los que no padecen enfermedad renal. Estas vasculopatías se encuentran en un $60 \%$ de las autopsias de estos pacientes, que pueden contribuir a alteraciones de la conducción, isquemia cardiaca, insuficiencia cardiaca congestiva y muerte ${ }^{3}$. La consecuencia de la pérdida de elasticidad vascular producto de estas calcificaciones ha resultado ser un importante predictor de morbimortalidad en pacientes urémicos ${ }^{8}$.

El tratamiento más aceptado en las calcificaciones es la paratiroidectomia subtotal, total y con autoimplante de tejido paratiroideo en el antebrazo. En el año 1991 Rothmund público un estudio que concluyo que la paratiroidectomia total con autoimplante, es la mejor opción, para estos pacientes, cuyas indicaciones más específicas son: progresión acusada de la osteítis fibrosa a pesar de un adecuado tratamiento médico, hipercalcemia persistente asociada con otros síntomas como hipertensión severa hiperfosforemia intensa, junto a evidencia histológica y radiológica de hiperparatiroidismo, prurito intenso intratable asociado con hiperparatiroidismo significativo, calcificaciones de tejidos blandos graves y persistentes asociadas a hiperfosforemia y evidencia de hiperparatiroidismo, necrosis cutánea y diseminada idiomática, osteopenia de vertebras, marcada deformidad esquelética y dolor óseo intenso $0^{9,10}$.
Una de las complicaciones habituales, que se pueden presentar en el post operatorio inmediato post paratiroidectomia, es la hipocalcemia, denominada "síndrome del hueso hambriento" secundario al rápido deposito de minerales en el hueso. Las estrategias para el tratamiento de la hipocalcemia postoperatoria, comprenden la administración de calcitriol antes de la cirugía, la monitorización estrecha de los niveles de calcio y el suplemento agresivo de calcitriol y calcio tras la cirugía 3 .

A continuación se presenta el caso clínico de una paciente con ERCT en diálisis peritoneal, que presentó calcificaciones metástasicas, asociadas a hiperparatiroidismo secundario.

\section{Objetivos}

1. Conocer la importancia de la pesquisa precoz del hiperparatiroidismo en pacientes en diálisis para evitar complicaciones posteriores.

2. Establecer intervenciones de enfermería en el paciente con alteraciones en el metabolismo fósforocalcio.

\section{Caso clínico}

Paciente de sexo femenino de 52 años portadora del síndrome de Sjogren, lupus eritematoso sistémico, trastorno depresivo, ERCT, osteoporosis secundaria, hiperparatiroidismo, miopatía secundaria, que ingresa a hemodiálisis en octubre de 2007, pero que luego de accesos vasculares disfuncionales, debe ingresar a peritoneo diálisis en el año 2008, hasta la actualidad, donde se encuentra en la modalidad diálisis peritoneal automatizada.

Hacia fines del año 2009 se plantea la necesidad de realizarle paratiroidectomia, debido a alteraciones en aumento de las pruebas de laboratorio especíicas, cintigrama compatible con tejido paratiroideo hiperfuncionante, y pesquisa (marzo 2011), de una masa abdominal asintomática, palpable de $10 \mathrm{~cm}$. infra umbilical, que no tiene relación con el túnel subcutáneo del catéter, por lo cual se realiza una tomografía axial computarizada de abdomen y pelvis con contraste, encontrándose en zona subcutánea un importante aumento de densidad de aspecto inflamatorio, que luego de descartar otra etiología se diagnostica como "calcificación metastásica abdominal". 
En abril del 2011 se realiza la paratiroidectomia total con autoinjerto en brazo derecho, cursando durante el periodo postoperatorio inmediato con hipocalcemia sintomática de $8,8 \mathrm{mg} / \mathrm{dl}$, la cual se manejó con bomba de infusión continua de calcio, con respuesta adecuada a ella, posteriormente se da el alta con controles semanales de calcio, y tratamiento de calcio y vitamina D. Sin embargo en mayo de 2011 presenta episodio de hipocalcemia sintomática con valor de calcio de $6,4 \mathrm{mg} / \mathrm{dl}$, la cual se manejó con hospitalización y calcio endovenoso entre otras medidas. En octubre de 2011 es hospitalizada por cuadro sintomático severo de hipercalcemia $(17,9 \mathrm{mg} / \mathrm{dl})$, el que se manejó en unidad de cuidados intensivos, con buena respuesta al tratamiento, a enero del 2012 presentaba una disminución del $50 \%$ de la masa abdominal. Actualmente permanece con controles seriados y estrictos, sin presentar nuevos episodios de hiper e hipocalcemia.

\section{Plan de cuidados:}

Los cuidados de enfermería se deberian orientar a la solución de los problemas reales o potenciales, poniendo énfasis en la prevención y educación constante.

1. Alto riesgo del mecanismo fósforo- calcio relacionado con disminución de la velocidad de filtración glomerular bajo del 15\%...

a. Valorar y educar en relación a la ingesta de alimentos ricos en fósforo y la importancia de la correcta administración de la terapia medicamentosa (quelantes del fósforo, análogos de vitamina $D$, calcitriol oral).

b. Educar sobre la relevancia de respetar la frecuencia, duración y eficiencia de la terapia dialítica.

c. Valorar e informar los exámenes mensuales específicos alterados, para establecer tratamiento y monitorizar los niveles de calcio serico, fósforo, producto calcio-fósforo, PTH.

d. Entrevistar al paciente y realizar exámen físico, para pesquisar signos y síntomas de calcificaciones metastásicas en cada sesión de diálisis.

e. Monitorizar la concentración de calcio en el líquido de diálisis y evaluar modificación según valores plasmáticos.

f. Establecer los cuidados preoperatorios en pacientes con indicación de hiperparatiroidectomia.
2. Alto riesgo de alteración de los niveles plasmáticos de calcio relacionado con extracción de la hormona paratiroidea.

a. Monitorizar los niveles plasmáticos de calcio constantemente y en caso necesario, según sintomatología derivar a consulta médica.

b. Realizar examen físico periódicamente y pesquisar alteraciones.

c. Reforzar educación en sintomatología relacionada con alteración de los niveles de calcio e importancia en la ingesta de suplementos.

\section{Alto riesgo de fracturas espontáneas relacionado con desmineralización ósea.}

a. Buscar manifestaciones de fracturas patológicas: dolor óseo, limitación de la movilidad, hinchazón de los tejidos y piel circundante.

\section{Alteración de la nutrición: por defecto r/c patolo- gía de base}

a. Controlar ingestión de alimentos y líquidos y calcular el consumo diario de calorías, animando al paciente a participar en la planificación del menú, aconsejar ingesta de alimentos en compañía.

b. Aconsejar al usuario y familiares llevar un diario de alimentación, para registrar y estimar calorías ingeridas, electrolitos de interés (potasio, sodio, cloruros, magnesio, fósforo, calcio) y proteínas.

c. Recomendar: el consumo de comidas frecuentes, en poca cantidad y el uso de condimentos permitidos hierbas o especias: ej: ajo, cebolla, pimienta, perejil, cilantro y limón.)y el cuidado oral permanente.

d. Reforzar el cumplimiento de la terapia nutricional ( oral y escrita) indicada por especialista.

e. Controlar datos de laboratorio (fósforo, calcio proteínas séricas, albúmina, hematocrito, hemoglobina, ferritina) y derivar en forma oportuna a especialistas.

\section{Deficit de conocimiento (necesidad de aprendiza- je) r/c su situación, pronóstico, tratamiento, auto- cuidado y necesidades.}

a. Comprobar el grado de ansiedad o temor y alteración de los procesos del pensamiento, animándole y dándole oportunidad para preguntar sus dudas y derivar a especialista en caso necesario.

b. Revisar en conjunto con el usuario la enfermedad específica, su pronóstico y las posibles complica- 
ciones con términos claros y concisos, repitiendo periódicamente y actualizando la información como sea necesaria.

c. Reconocer que algunos sentimientos y conductas en el usuario son normales durante la evolución del tratamiento.

d. Subrayar la importancia de leer las etiquetas de todos los productos (alimentos y bebidas, fármacos sin receta) y no tomar medicamentos ni complementos de fitoterapia sin autorización previa del facultativo.

e. Insistir en la importancia de fijar y cumplir un horario para terapia dialítica, medicación y hábitos alimentarios.

f. Frente a posibles modificaciones de la terapia dialítica, satisfacer las necesidades educativas del usuario, entregándolas de manera comprensible y reiteralas cuando las solicite.

\section{Conclusiones}

Los pacientes en diálisis son vulnerables de presentar variadas complicaciones, que van en desmedro de su calidad de vida. Las alteraciones óseas son una de ellas, presentándose en forma lenta y progresiva, donde la prevención, pesquisa y derivación oportuna es una tarea imprescindible del equipo de salud.

En estos pacientes, se debe tener en cuenta que coexisten múltiples factores de riesgo, los cuales en conjunto deben ser abordados precozmente para realizar intervenciones que puedan prevenir las complicaciones que a largo plazo presentaran riesgos de mayor magnitud.

Al evaluar el caso clínico proponemos como enfermeras especialistas en el cuidado del paciente renal, establecer en los centros de diálisis un control semestral por enfermera, independiente de las sesiones de diálisis, donde podamos pesquisar con mayor privacidad y autonomía, las necesidades particulares de cada uno de estos pacientes contribuyendo precozmente a una mejor adherencia al tratamiento y retardo progresivo de las complicaciones propias de esta enfermedad.

El profesional de enfermería es una de las personas más cercanas, al paciente en terapia de sustitución renal y en el recae gran parte de la tarea educativa, que debe ser permanente y enfocada al tratamiento, autocuidado y satisfacción de las necesidades individuales de estos usuarios, la que se verá reflejada posteriormente, en una mejor calidad de vida propia y de su entorno.
Recibido: 13 Junio 2013

Revisado: 30 Junio 2013

Modificado: 20 Agosto 2013

Aceptado: 22 Agosto 2013

\section{Bibliografía}

1. Flores J, Alvo M, Borja H, Morales J, Vega, J, Zuñiga $C$, et al. Complicaciones sistémicas en enfermedad renal crónica. Metabolismo oseomineral en el paciente renal. Revista Médica de Chile 2009: 168-172.

2. Fernández $\mathrm{H}$, Patricio J, Oliveira $P$, Oliveira A, Faria T, Pereira J, et al. Calcificaçiones metastáticas detectada en cintigrafia óssea. Acta Médica Portuguesa 2010; 23: 731-734.

3. Nissenson A, Fine R. Manual de Diálisis. Demografía: 3-20, 0steodistrofía Renal. España: Elsevier, 2009: 965-986.

4. Daugirdas J, Manual de diálisis. En: Van Stone J. Y Daugirdas T. Bases fisiológicas y modelo cinético de la urea. $4^{a}$ edición. ed. Lippincot, Williams \&Wilkins.2007.

5. Weinstein $L$, Swartz MN. Pathologic properties of invading microorganismos. En: Sodeman WA, Sodeman LA, editores. Pathologic physiology: mechanisms of disease. Philadelphia: Saunders, 1974: 457-472.

6. Caudrillier A, Mentaverri R, Brazier M, Kamel S, Massy Z. Calcium- sensing receptor as a potencial modulator of vascular calcification in chronic kidney disease. J Nephrol 2010; 23: 17-22.

7. Verdalles $U$, De la Cueva $P$, Verde $E$, García de Vinuesa S, Goicoechea M. Mosse A, et al. Calcifilaxis: complicación grave del síndrome cardio-metabólico en pacientes con enfermedad renal crónica terminal. Órgano Oficial de la Sociedad Española de Nefrología 2008:32-36.

8. López Oliva M, Del Castillo D, Sánchez J. Alteraciones del metabolismo óseo mineral en la enfermedad renal crónica del paciente trasplantado. Organo Oficial de la Sociedad Española de Nefrología: 2009: 31-37. 
9. Junyent $M$, Martínez $M$, Borrás $M$, Betriu $A$, Coll $B$, Craver $L$, et al. Utilidad de las técnicas de imagen y biomarcadores en la predicción del riesgo cardiovascular en pacientes con enfermedad crónica terminal en España: Proyecto Nefrona. Organo Oficial de la Sociedad Española de Nefrología 2010:119-126.
10. Heitz U, Horne M. Fluidos, electrolitos y equilibrio ácido-base. En: Alteraciones del Calcio. España: 2006: 128-140.

11. Perez J, Gabrielli M, Born N. Evaluación prospectiva de paratiroidectomias en el tratamiento del hiperparatiroidismo secundario. Revista chilena de cirugía 2009: 15-20. 